\title{
Depinning exponents of the driven long-range elastic string
}

\author{
Olaf Duemmer* and Werner Krauth \\ CNRS-Laboratoire de Physique Statistique \\ Ecole Normale Supérieure \\ 24 rue Lhomond, 75231 Paris Cedex 05, France
}

\begin{abstract}
We perform a high-precision calculation of the critical exponents for the long-range elastic string driven through quenched disorder at the depinning transition, at zero temperature. Large-scale simulations avoid finite-size effects and improve accuracy. We explicitly demonstrate the equivalence of fixed-velocity and fixed-driving-force simulations. The roughness, growth, and velocity exponents are calculated independently, and the dynamic and correlation length exponents are derived. The critical exponents satisfy known scaling relations and agree well with analytical predictions.
\end{abstract}

Driven elastic manifolds in disordered media model a multitude of physical systems ranging from cracks advancing in a solid in response to an external strain field [1] to charge density waves [2], vortices in type-II superconductors in an electric field [3] , and to magnetic domain walls in an external magnetic field [4]. These widely studied out-ofequilibrium systems undergo dynamic phase transitions [5] as a function of the external driving force (strain, electric or magnetic field, etc.). At driving forces below the depinning threshold $\left(f<f_{c}\right)$, the disorder pins the manifold so that its center-of-mass velocity vanishes at large times. Above the depinning threshold $f_{c}$, avalanches advance the manifold with finite center-of-mass velocity. At the depinning threshold (for $f \rightarrow f_{c}^{+}$), the center-of-mass velocity in the long-time limit goes to zero algebraically, whereas the typical length, width and duration of the avalanches diverge. This critical divergence is characterized by universal scaling exponents.

Most simply, advancing crack fronts are modeled by the dynamics of an elastic string driven through quenched disorder (the inhomogeneous material). On that level, elasticity theory combined with a reasonable energy dissipation mechanism can be reduced to an effective equation of motion for the crack front [1, 6, 7]. The resulting elastic energy kernel is long ranged, i.e. falls of as the squared inverse distance. The same long-range elastic energy kernel is found in the wetting problem of an advancing contact line [8].

In the last fifteen years, well-controlled experiments have been designed to measure universal scaling exponents $[9,10,11,12,13]$. But the experimental precision available does not yet allow to give definite answers on questions about universality. In addition, experimental results on the roughness exponent of contact lines are inconsistent with theoretical predictions. However, a long-standing controversy on numerical values of the roughness exponent has recently been resolved $14,15,16,17,18,19,20]$.

In this paper, we perform a numerical simulation of the depinning phase transition of the long-range string driven through quenched disorder, with the aim of computing the critical exponents to high precision. Numerical simulations of this model are non-trivial because of large finite-size effects, caused by the unusually large value of the finite-sizescaling exponent.

The discrete long-range elastic string is represented by a vector of integer heights $\left\{h_{1}, \ldots, h_{L}\right\}$ with periodic boundary conditions. The string follows discrete quasi-static dynamics while being driven through a discrete disorder (cf [21]). The long-range elastic force acting on a string element $i$ is given by

$$
f_{i}^{\text {elastic }}=\sum_{j \neq i}^{L} \frac{h_{j}-h_{i}}{|j-i|^{2}} .
$$

Even though the sum contains $\sim L$ terms for each of $L$ sites, all forces on all sites can be computed in $\sim L \log L$ operations using a fast-Fourier-transform (FFT) algorithm.

The quenched disorder generates a random force $\eta$ :

$$
f_{i}^{\text {random }}=f+g \eta_{i}\left(h_{i}\right) .
$$

While $f$ is a constant external field, the random position-dependent force $\eta$ is drawn from a binomial distribution: $\eta$ equals plus one with probability $p$, and minus one with probability $1-p$. The coupling $g$ [22] controls the Larkin length at which the elastic and disorder forces balance.

The random force results in a net external driving force of $f+g(2 p-1)$. The parameter $f$ can be adjusted to keep constant the otherwise fluctuating center-of-mass velocity $v$ of the string. But we use fluctuating-velocity/fixed-force simulations throughout this paper, unless stated otherwise. $p$ is the control parameter of the dynamic phase transition: its distance to the critical value $p_{c}$ (the depinning threshold) controls the large-scale properties of the dynamics, the 


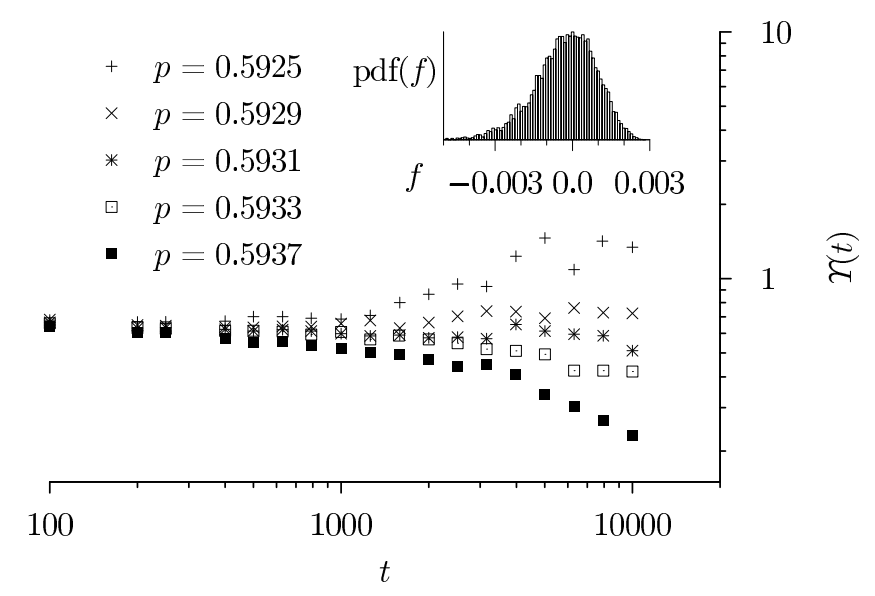

FIG. 1: At the depinning threshold $p_{c}$, the ratio $\Upsilon(t)=w /[t v(t)]$ is constant in time, as shown by scaling arguments (for $p<p_{c}, \Upsilon(t \rightarrow \infty) \rightarrow \infty$ because the velocity vanishes, and for $\left.p>p_{c}, \Upsilon(t \rightarrow \infty) \rightarrow 0\right)$. The inset shows fluctuations around zero of the external force $f$ for a simulation with fixed $p_{c}$ and fixed velocity: one percent of all sites move at each time step.

divergence of the correlation length and the algebraic decay of the mean velocity $v$ [23]. The skewed binomial distribution of random forces corresponds to random-field frozen disorder, as opposed to random-bond disorder.

The microscopic dynamics of the string is given by the following discrete dynamical rule:

$$
h_{i}(t+1)-h_{i}(t)=v_{i}(t)=\left\{\begin{array}{ll}
1 & \text { if } f_{i}^{\text {elastic }}+f_{i}^{\text {random }}>0 \\
0 & \text { otherwise }
\end{array} \quad t=0,1, \ldots .\right.
$$

This rule does not allow for backward motion, as is justified by the no-return theorem [24].

Very large system sizes are crucial in order to calculate the critical exponents with sufficient precision. Consider for example the velocity exponent $\beta$, defined as $v \sim\left(p-p_{c}\right)^{\beta}$. For it to have an accuracy of $\delta \beta=0.01$ requires a resolution of $\delta p_{c}=10^{-3}$ in the critical force 25]. This resolution can only be obtained if the finite-sample-size fluctuations of the critical force $\sigma_{p_{c}}$ are small enough. From a finite-size-scaling analysis one finds $\sigma_{p_{c}}(L)=0.65 \times L^{-1 / \nu_{\mathrm{FS}}}, \nu_{\mathrm{FS}}=1.62$ (data not shown), and demanding that $\sigma_{p_{c}}<10^{-3}$ gives the minimum system size $L_{\min } \approx 10^{5}$.

The discretized model, with its coarse and low-cost description of microscopic dynamics, can be simulated for sizes up to $L=2^{20} \approx 10^{6}$, more than three orders of magnitude larger than for earlier discrete [17, 18] and continuum simulations [26].

To benefit from the high speed of the FFT algorithm, we use parallel updating: The elastic force on all string elements is calculated. Then, the dynamic rule (equation (31) ) is applied simultaneously to all sites. Finally, the random force is updated for those sites that have stepped forward. In this way a single vector of length $L$ suffices to store the random force, avoiding storage problems even for very large system sizes. The parallel updating leads to a fluctuating mean velocity $v$, whereas the external driving force is kept constant via the fixed control parameter $p$.

The depinning threshold $p_{c}$ is determined by adapting a technique known from absorbing-state dynamic phase transitions 27]: we start the simulation for fixed $p, f=0$ from a flat line at $t=0$, and monitor the ratio $\Upsilon$ of string width $w(t) \equiv\left[(1 / L) \sum_{i}^{L}\left(h_{i}-\langle h\rangle\right)^{2}\right]^{1 / 2}$ to decaying center-of-mass velocity $v(t) \equiv(1 / L) \sum_{i}^{L} v_{i}(t)$ times time $t$ :

$$
\text { At threshold : } \quad v(t) \sim \frac{w(t)}{t}=t^{\zeta / z-1}, \quad \Longrightarrow \quad \Upsilon(t) \equiv \frac{w(t)}{t v(t)} \sim \text { constant. }
$$

To understand why the velocity at threshold is given by the width divided by time, we remark that, intuitively, an avalanche takes time $t$ to advance a distance equal to its width $w(t)$. Moreover, at threshold, the motion of the string is solely due to avalanches, hence the mean velocity is given by $v(t)=w(t) / t$. It follows that the ratio $\Upsilon(t)$ is constant at threshold. Away from threshold it either grows to infinity (for $p<p_{c}$ ) or decays to zero (for $p>p_{c}$ ), providing a very sensitive indicator for the depinning threshold.

The main plot in figure 1 illustrates the sensitivity of $\Upsilon(t)$ to tiny changes in $p-p_{c}$, which in turn enables a very precise localization of the critical threshold $p_{c}=0.5931(2) . p_{c}$ can alternatively be extracted from a power-law fit of the steady-state mean velocity $\left(v \sim\left(p-p_{c}\right)^{\beta}\right.$, see figure 5). 


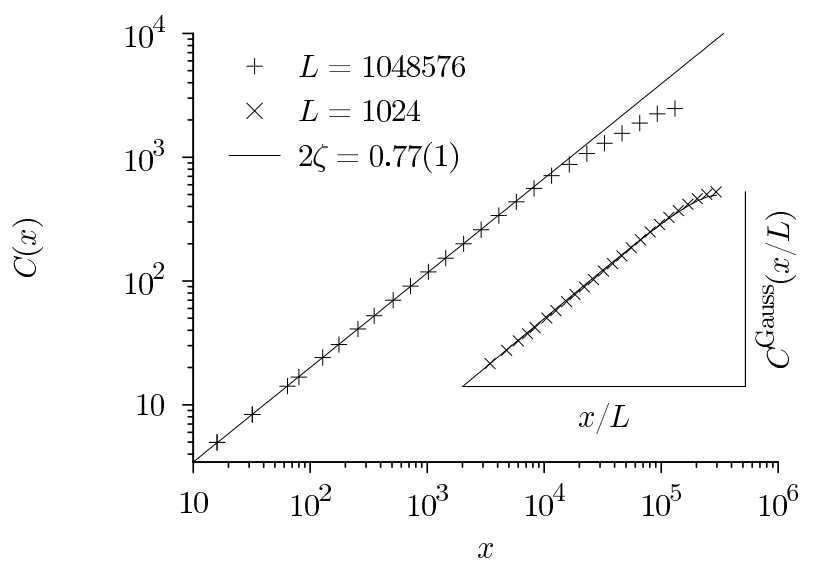

FIG. 2: Roughness exponent $\zeta$. At short distances, the two-point correlation function scales as $C(x) \propto x^{2 \zeta}$. A linear fit gives $\zeta=0.385(5)$ (average taken over pinned configurations exactly at $p=p_{c}$ ). The inset compares correlation-function data (for $L=1024)$ with the Gaussian model of (eq. 4).

There has been debate about the equivalence between simulations with fluctuating velocity/fixed force on the one hand and with fixed velocity/fluctuating force on the other. In the thermodynamic limit, one expects them to be equal because the fluctuations of the center-of-mass velocity vanish on large enough length scales. In experimental or numerical systems, the center-of-mass velocity fluctuates only on scales smaller than the correlation length. In our very large simulations, we are able to check the equivalence between the two approaches: in addition to the fluctuating-velocity simulations in the rest of this paper, we perform a fixed-velocity simulation by adjusting the parameter $f$ at each time step such that a very small but fixed proportion of sites move, thus automatically tuning the system to the depinning threshold. The inset of figure 1 shows the minute fluctuations of the external force $f$ around a zero mean value in a simulation where the external force is chosen at each time-step such that one percent of sites are made to move [28], at $p=p_{c}$. This demonstrates that $p_{c}$ is indeed the critical point, and that the two types of simulations are equivalent.

The roughness exponent $\zeta=0.388$ [20] characterizes the self-affine spatial structure of the string at depinning, and the scaling properties of its average width $w$ (see also [29]). As a check for the discrete model, we compute this exponent from the short-distance behaviour of the two-point correlation function $C(x) \equiv\left\langle(h(0)-h(x))^{2}\right\rangle \sim x^{2 \zeta}$. Averaging over pinned configurations exactly at the depinning threshold $p=p_{c}$, we confirm earlier results $(\zeta=0.385(5)$, see figure 2).

The correlation function $C(x)$ can be fitted over the whole interval $x \in[0,0.5 L]$ using the Gaussian model [30]: suppose the random string configuration $h(x)$ is decomposed into Fourier modes

$$
h(x)=\sum_{n} a_{n} \cos (2 \pi n x / L)+b_{n} \sin (2 \pi n x / L),
$$

where $a_{n}$ and $b_{n}$ are Gaussian random variables with variance $\sigma_{n}^{2} \propto 1 / n^{1+2 \zeta}$. The resulting two-point correlation function is given by $C^{\text {Gauss }}(x)=4 \sum_{n} \sin ^{2}(\pi n x / L) / n^{2 \zeta+1}$, with the following expansion for small $x / L$ [30] (note the difference between the roughness exponent $\zeta$ and the Riemann zeta-function $\zeta_{\mathrm{R}}$ ):

$$
\begin{aligned}
C^{\text {Gauss }}(x)=\text { constant }\left\{-\left(\frac{x}{L}\right)^{2 \zeta} 2^{-1+2 \zeta} \pi^{2 \zeta} \Gamma(\right. & -2 \zeta) \sin \frac{\pi(2 \zeta+1)}{2} \\
& \left.+\left(\frac{x}{L}\right)^{2} \frac{2 \pi^{2}}{2 !} \zeta_{\mathrm{R}}(2 \zeta-1)-\left(\frac{x}{L}\right)^{4} \frac{8 \pi^{4}}{4 !} \zeta_{\mathrm{R}}(2 \zeta-3)+O\left[\left(\frac{x}{L}\right)^{6}\right]\right\} .
\end{aligned}
$$

This function is used in the inset of figure 2 to fit the correlation function data obtained with the exact algorithm [31] for smaller systems. The fit remains excellent even for large $x / L$. 


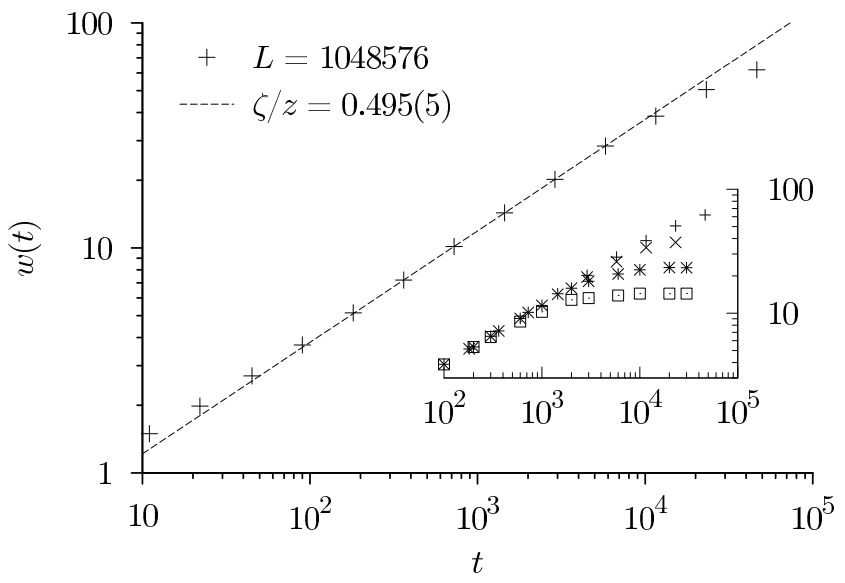

FIG. 3: Growth exponent $\zeta / z$. The algebraic growth of the average width $w(t) \sim t^{\zeta / z}$, at the depinning threshold $\left(p=p_{c}\right)$ and after initial relaxation effects $(t \sim 100)$, gives the growth exponent $\zeta / z=0.495(5)$. Inset: large finite-size effects as width levels off at $w_{L} \sim L^{\zeta}$, for $L=\left\{2^{14}, \ldots, 2^{20}\right\}$.

The dynamic exponent $z$ was previously estimated for discrete extremal models of limited size [17, 18]. We determine it via the growth exponent $\zeta / z$, which controls the growth of the average width with time $w(t) \sim t \zeta / z$. This algebraic growth law results from the two scaling laws for the width and the time in terms of the correlation length $\xi: w \sim \xi \zeta$ and $\xi \sim t^{1 / z}$. We start at $t=0$ from a flat line, at the critical point $p=p_{c}$, and monitor the growth of the average width. Figure 3 shows how, after an initial relaxation phase [32], the width grows algebraically with the growth exponent $\zeta / z=0.495(5)$, implying $z=0.770(5)$ for the dynamic exponent. The width $w(t)$ is also affected by large finite-size effects. The algebraic growth regime is limited by the width of the entire string $w_{L} \sim L^{\zeta}$. The inset of figure 3 demonstrates how $w(t)$ saturates at the system-size-dependent limit $w_{L}$. The plots for different system sizes $L$ can be superposed by plotting $w(t) L^{-\zeta}$ versus $t L^{-z}$ (not shown).

Another way of determining the dynamic exponent $z$ is to analyze the time-dependent structure factor $S(q, t) \equiv$ $\langle h(q, t) h(-q, t)\rangle$ with the Fourier transform of the height given by $h(q) \equiv \sum_{n=0}^{L-1} \exp (\mathrm{i} q n) h_{n}$ and $q \equiv 2 \pi k / L$, where $k \in\{0, \ldots, L-1\}$ is the mode number. The structure factor allows us to illustrate both the dynamic growth of the interface and its self-affine structure in space-time [33]:

$$
S(q, t) \sim q^{-1-2 \zeta} \Phi\left(q^{z} t\right) \quad \text { with } \quad \Phi(y)=\left\{\begin{array}{l}
y^{\frac{1+2 \zeta}{z}}, y \rightarrow 0 \\
\text { constant }, y \rightarrow \infty
\end{array}\right.
$$

Figure 4 illustrates the convergence of the different Fourier modes $S(q, t)$ to their steady-state value $\sim q^{-1-2 \zeta}$ after a relaxation time $q^{-z}$. The small-time behavior $S(q, t) \sim t^{(1+2 \zeta) / z}$ provides the dynamic exponent, and we confirm the numerical value $z=0.77(1)$, albeit with lower precision than in our analysis using $w(t)$. The inset of figure 4 plots $S(q, t) q^{1+2 \zeta}$ versus the dimensionless quantity $q^{z} t$. It shows the universal scaling form $\Phi$, and demonstrates the self-affine link between time $t$ and (inverse) space $q$, via the dynamic exponent $z$.

The velocity exponent $\beta$ controls the power-law decrease of the mean velocity with the distance to the critical threshold: $v \sim\left(p-p_{c}\right)^{\beta}$. It is obtained from the center-of-mass velocity $v$ in the steady-state regime (see figure 5). From a fit at fixed $p_{c}$, we obtain $\beta=0.625(5)$. The data of the steady-state velocity can also be fitted to obtain the threshold $p_{c}$, as shown in the inset of figure 5. Only for $p=p_{c}$ is there a straight line on the $\log -\log$ plot. For $p \neq p_{c}$, a clear non-zero curvature emerges.

Note that it is quite difficult to obtain a precise estimate for $\beta$, again because of large finite-size effects. Trying to calculate $\beta$ on medium-size systems one faces the problem of a significantly reduced critical scaling regime. It manifests itself already in the short-range elastic model [26], and is even more pronounced on long-range elastic interfaces of intermediate size (data not shown). Our very large systems allow to obtain satisfying accuracy. 


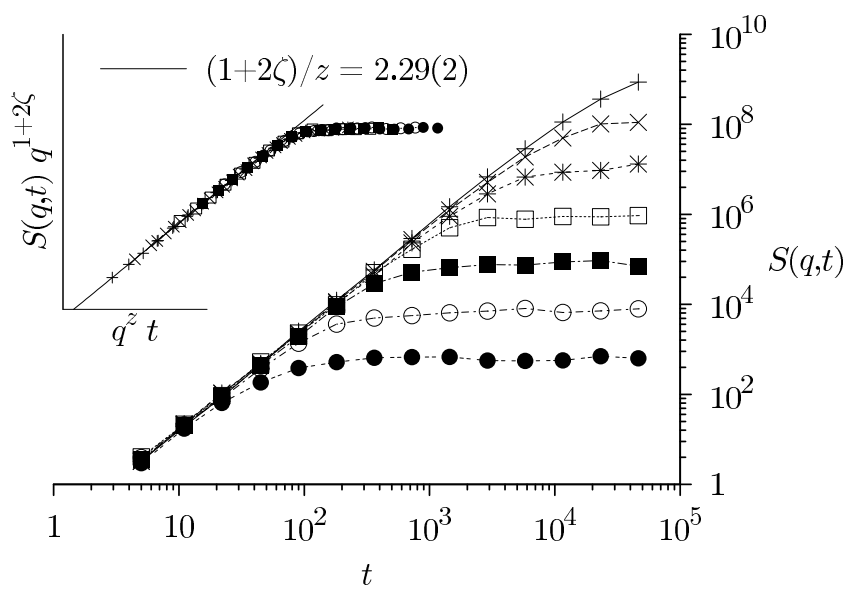

FIG. 4: Time-dependent structure factor $S(q, t)$. Different Fourier components [from $(+) q=3 \times 10^{-6}$ to $\left.(\bullet) q=10^{-2}\right]$ take a relaxation time of $q^{-z}$ to reach their steady-state value of $q^{-1-2 \zeta}$. Inset: the small-time behaviour of the universal scaling function gives a further estimate of the dynamic exponent $z=0.77(1)$.

Our value for the velocity exponent yields an independent check of the growth exponent $\zeta / z$, via two scaling relations that reduce the set of four critical exponents to two independent ones: the statistical tilt symmetry [34] links the roughness exponent to the correlation length exponent $\nu=1 /(1-\zeta)$, and a further scaling relation links all four exponents $\beta=\nu(z-\zeta)$ 34]. Using these two equalities and values of $\zeta=0.385(5)$ and $\beta=0.625(5)$ we converge on a value for the growth exponent of $\zeta / z=0.500(5)$. These numerical values, our final result, reflect our numerical measurements and the constraints of the scaling relations, which are finally used to deduce the dynamic exponent $z=0.770(5)$, and the correlation length exponent $\nu=1.625(10)$.

In conclusion, we have numerically studied the non-equilibrium depinning phase transition of the long-range elastic string driven through quenched disorder. The values obtained for the critical exponents satisfy scaling relations and agree with analytical predictions from functional RG [14, 15, 16]. For completeness we recall the one- and two-loop $\left(\epsilon, \epsilon^{2}\right)$ results: $\zeta_{\epsilon}=0.33, \zeta_{\epsilon^{2}}=0.47 ; \beta_{\epsilon}=0.78, \beta_{\epsilon^{2}}=0.59 ; z_{\epsilon}=0.78, z_{\epsilon^{2}}=0.66 ; \nu_{\epsilon}=1.33, \nu_{\epsilon^{2}}=1.58$. On the experimental side, it will be interesting to understand why exactly the roughness and growth exponents calculated in this paper agree with those measured in a recent crack front experiment [35], whether they can also be seen in wetting experiments, and more generally, whether universality can be confirmed as the physical mechanism unifying these diverse phenomena.

We thank D. Bonamy, E. Bouchaud, H. Chaté, A. Kolton, P. Le Doussal, S. Guibert, L. Ponson, A. Rosso, and K. J. Wiese for stimulating discussions.

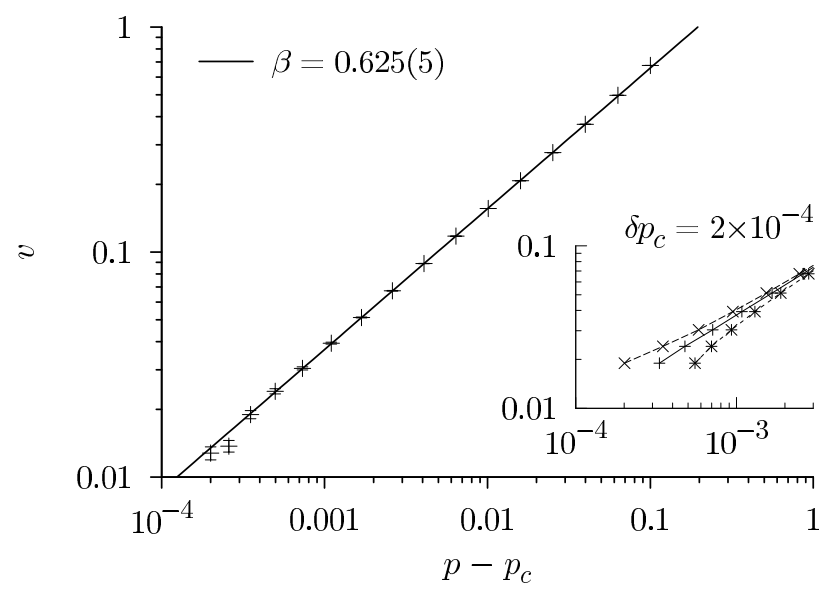

FIG. 5: Velocity exponent $\beta$. The steady-state mean velocity as a function of the control parameter $v \sim\left(p-p_{c}\right)^{\beta}$ gives the velocity exponent: $\beta=0.625(5)$. Inset: slightly wrong values of $p_{c}$ lead to non-zero curvature $\left((\times) p_{c} \rightarrow p_{c}-\delta p_{c},(*) p_{c} \rightarrow\right.$ $\left.p_{c}+\delta p_{c}\right)$. 
* Electronic address: duemmer@lps.ens.fr

$\dagger$ Electronic address: krauth@lps.ens.fr

[1] H. Gao, J. R. Rice, J. Appl. Mech. 56, 828 (1989).

[2] G. Grüner, Rev. Mod. Phys. 60, 1129 (1988).

[3] G. Blatter et al., Rev. Mod. Phys. 66, 1125 (1994).

[4] S. Lemerle et al., Phys. Rev. Lett. 80, 849 (1998).

[5] D. S. Fisher, Phys. Rev. B 31, 1396 (1985).

[6] S. Ramanathan, D. Ertaş, D. S. Fisher, Phys. Rev. Lett. 79, 873 (1996).

[7] J. P. Bouchaud, E. Bouchaud, G. Lapasset, J. Planès, Phys. Rev. Lett. 71, 2240 (1993).

[8] J. F. Joanny, P. G. De Gennes, J. Chem. Phys. 81, 552 (1984).

[9] S. Moulinet, C. Guthmann, E. Rolley, Eur. Phys. J. E, 8, 437 (2002).

[10] E. Schäffer, P. Wong, Phys. Rev. E, 61, 5257 (2000).

[11] A. Prevost, E. Rolley, C. Guthmann, Phys. Rev. B, 65, 064517 (2002).

[12] K. L. Måløy, S. Santucci, J. Schmittbuhl, R. Toussaint, Phys. Rev. Lett. 96, 045501 (2006).

[13] L. Ponson, D. Bonamy, E. Bouchaud, Phys. Rev. Lett. 93, 035506 (2006).

[14] O. Narayan, D. S. Fisher, Phys. Rev. B, 48, 7030 (1993).

[15] D. Ertaş, M. Kardar, Phys. Rev. E, 49, R2532 (1994).

[16] P. Chauve, P. Le Doussal, K. J. Wiese, Phys. Rev. Lett. 86, 1785 (2001); P. Le Doussal, K. J. Wiese, P. Chauve, Phys. Rev. B, 66, 174201 (2002).

[17] A. Tanguy, M. Gounelle, S. Roux Phys. Rev. E, 58, 1577 (1998).

[18] J. Schmittbuhl, S. Roux, J. P. Vilotte, K. J. Måløy, Phys. Rev. Lett. 741787 (1995).

[19] S. Ramanathan, D. S. Fisher, Phys. Rev. B, 58, 6026 (1998).

[20] A. Rosso, W. Krauth, Phys. Rev. E, 65, 025101(R) (2002).

[21] H. Leschhorn, T. Nattermann, S. Stepanow, L. H. Tang, Ann. Physik, 6, 1 (1997).

[22] We set $g=1$ throughout this paper.

[23] Surprisingly, setting $p=1 / 2$ while letting $f$ approach the critical value $f_{c}=g\left(2 p_{c}-1\right)$ is not equivalent to our procedure. The discreteness of the model entails in this case that the asymptotic velocity does not approach zero continuously as $f \rightarrow f_{c}$, it rather drops discontinuously from a value of order unity at $f>f_{c}$ to zero at $f \leq f_{c}$.

[24] A. A. Middleton, Phys. Rev. Lett. 68, 670 (1992). The no-return theorem states that any initially forward-moving string obeying dissipative dynamics and having a convex elastic energy function will never recede in the course of its motion.

[25] Taking differentials of $v \sim\left(p-p_{c}\right)^{\beta}$ implies $\delta \beta / \beta=-\delta p_{c} /\left(\left|p-p_{c}\right| \log \left|p-p_{c}\right|\right)$ and measuring the $\beta$-slope at $p-p_{c} \approx 0.01$ in figure 5 gives $\delta p_{c} \approx 10^{-3}$.

[26] O. Duemmer, W. Krauth, Phys. Rev. E, 71, 061601 (2005).

[27] H. Hinrichsen, Adv. Phys. 49, 815-958 (2000).

[28] We choose $v=0.01$ because this is the limit of statistical resolution of a system of size $L \approx 10^{6}$ (cf figure 5). The sequential updating of a single site per time step leads to the smallest possible velocity and keeps the system exactly at the depinning threshold. Used in previous medium-size simulations [17, 18], it becomes however too slow for large systems, since all elastic forces $(\sim L \log L)$ have to be recalculated each time a single site advances.

[29] D. Vandembroucq, S. Roux, Phys. Rev. E, 70, 026103 (2004).

[30] A. Rosso, R. Santachiara, W. Krauth, J. Stat. Mech. Theory E, L08001 (2005); R. Santachiara, A. Rosso, W. Krauth, cond-mat/0611326.

[31] A. Rosso, W. Krauth, Phys. Rev. B, 65, 012202 (2001).

[32] A. B. Kolton, A. Rosso, E. V. Albano, T. Giamarchi, Phys. Rev. B, 74, 140201 (2006).

[33] A. L. Barabási, H. E. Stanley, Fractal Concepts in Surface Growth Cambridge University Press, Cambridge (1995).

[34] T. Nattermann, S. Stepanow, L. H. Tang, H. Leschhorn, J. Phys. (France) II 2, 1483 (1992).

[35] D. Bonamy, L. Ponson, S. Prades, E. Bouchaud, C. Guillot, Phys. Rev. Lett. 97, 135504 (2006). 